\title{
Composite Complex Technical Solutions as a Necessary Basic Activating Foundation for Creating Combined Software Products-Equivalents of Complex System Integrative Inventions
}

\author{
Konstantin A. Piliugin, Ph.D. \\ Voronezh, Russian Federation
}

Due to the introduction into everyday practice of various kinds of combined software products, usually including many interrelated elements not interconnected in early design versions, there was a real need to create composite and complex technical solutions where all elements previously not interconnected form a composite complex technical solution.

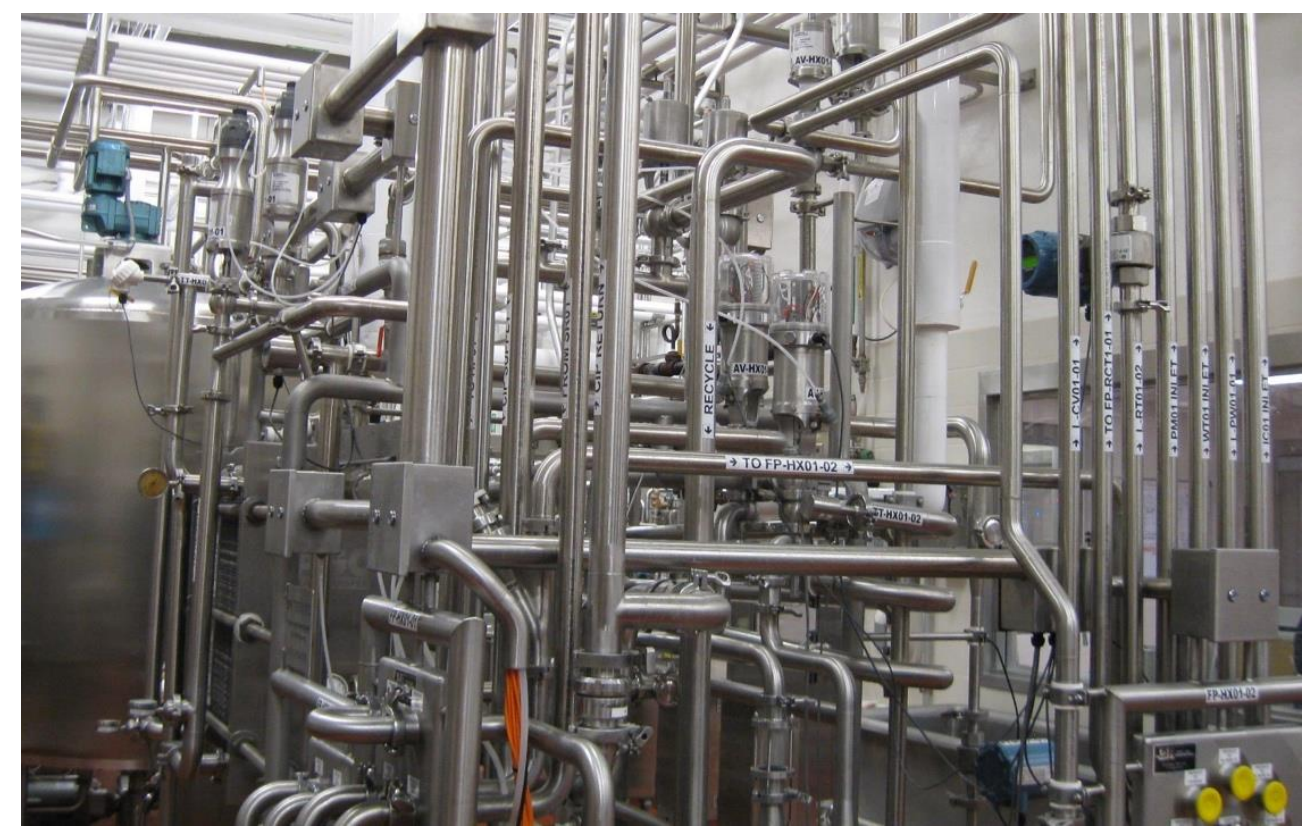

Figure 1. An example of a composite complex technical solution that includes combined software products, aligned with complex system integrative inventions in the field of environmentally friendly and fully automated dairy production. 
Almost complete transition of the economies of developed countries to innovative methods of planning and management dictates new criteria for the qualification of technical solutions used.

Gradually, any kind of production in such conditions, in order to maintain the necessary level of competitiveness - shall transform and optimize its technological and commercial assets to achieve the level of so-called smart production.

But it does not make much sense to create production, for production, without a serious well thought out strategic framework, including in terms of the most effective information tool for attracting partners and customers, such as web sites with a dynamic information system.

Today, more and more attention is paid to this process as part of smart production, including parallel creation of appropriate software techniques and tools, including, of course, three-dimensional graphics elements that carry more and more information containing data of innovative tools, equipment and materials used.

Taking into account the fact that, for example, in the USA, new patent legislation has already been adopted and introduced, it makes sense to dwell in greater detail on the feasibility of including in these information materials on the latest developments used in the advertised products and which can be qualified for compliance with the four basic signs of invention. This would be, first of all, the criterion of non-obviousness of the invented technical solution.

Of course, in such situations, for commercial reasons, it is important to preserve all possible levels of protection of the rights to the original intellectual property, even in cases when reliable protection has not yet been created and the product is already being delivered.

What kind of information and in what form can bring the level of information able to form a positive perception of the new product and all information related thereto, to concerned parties?

First of all, such information may include data on fundamentally new materials used in a new innovative product and their combinations with traditional materials that are quite well known in the market.

Of course, the disclosure of all details and features of the application must be approached with a known and sufficient degree of caution, since violation of the criteria of absolute novelty may close the way to patenting a product or its components. 
A huge role in ensuring the proper technical level of new products and their consumer qualities is played by the presence of composite materials therein, especially carbon-carbon compositions.

Innovative composite materials are usually combined with innovative manufacturing technology, also in terms of smart production, and the most valuable quality of such smart production is the capability to monitor quality in real-time at all stages of the production process.

Finally, the nature of information presentation, including on websites, is influenced and affected by new and modernized consumer standards as well as new environmental standards and their perception in each of the specific areas of the consumer market.

\section{Availability of fundamentally new materials}

Fundamentally new materials today can come in a wide variety of types and purposes and can be used in innovative products for a variety of reasons.

One of the most important aspects of the search for and use of fundamentally new structural materials is the need to use processing equipment with digital software control to manufacture parts and elements of a new product.

This equipment has a place for new structural tool materials and alloys, and especially cutting materials of increased hardness and durability.

Recently, metal-ceramic materials and their variants have become especially popular in this area.

The use of such materials significantly increases the reliability and accuracy of metalcutting tools, as well as equipment performance.

With the emergence of 3D-injection molding machines, various plastics and their ceramic equivalents are increasingly required for construction materials.

Variance can be very broad, and we can talk about a variety of final products, significantly differing in many technical characteristics, including mass and weight characteristics, as well as practical areas of application. 


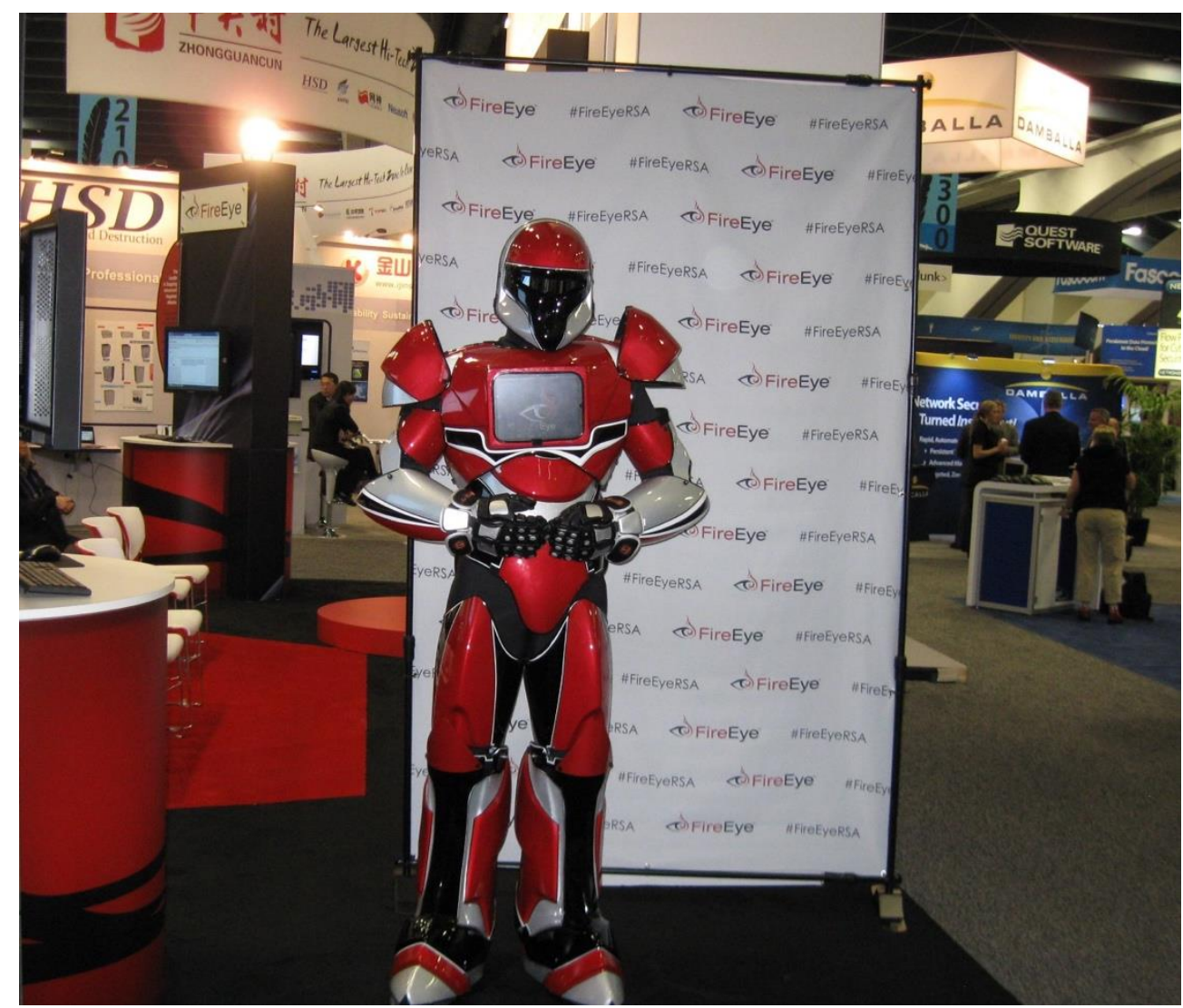

Figure 2. An example of the use of fundamentally new materials in fire protection demonstrated at the international exhibition of protective technologies and materials. This type of information does not reveal production, technological, constructive and operational secrets and at the same time allows us to bring all the winning parameters and capabilities of new products to potential customers.

As is known from the patent legislation, just the replacement of the used construction material with a new one, or even a fundamentally new one, is not an invention.

On the other hand, such a simple replacement is not always possible at all. Complete replacement of the material requires other additional technical solutions, including both schematic and component ones.

As a rule, in combination with the fundamental solutions on replacement of the material and on changes in the product properties and parameters arising from changes in the properties of the new material, a comprehensive technical solution arises, and its main distinguishing 
features form compliance of the invention with criteria, including on the basis of nonobviousness of the technical solution for the specialist of the average qualification in this area.

Most often, if a new product is developed, manufactured and tested in one working group or in a start-up team, patent search and qualification of a technical solution for compliance with signs can be carried out only after all production test cycles and final qualification tests of the actual technical feature of a new product.

If a new product includes a controlling and monitoring processor or a group of processors, then the nature of the search by the proposed invention expands, and the search pattern changes, just like the invention structure.

The invention scheme takes the following form - design, system, program, and method.

Thus, novelty of the distinctive features of an innovative product consists of several mutually dependent attributes relating to various elements of the working characteristic.

Particular interest in this process of integrative identification of new technical solutions is paid to the use of fundamentally new structural materials and technological solutions, emulsions and aerosols.

The method of presenting the information of these novelties to potential users of these products today is largely determined by the level of information load of the web product of the developer and manufacturer of this product.

This is because in principle, in accordance with the patent laws of the United States and other countries with innovative economies, simply replacing a material, even with one which is the newest and most advanced, is not an invention. Correct presentation of technological and constructive gains on the website without violating the product novelty, allows the maintaining of the principle of novelty and at the same time brings all the information required for decisionmaking to the attention of parties potentially interested in an innovative product.

The line between fundamentally new materials and composite materials can be quite narrow, but it still makes sense to allocate a separate place for composite materials.

\section{Presence of composite materials}




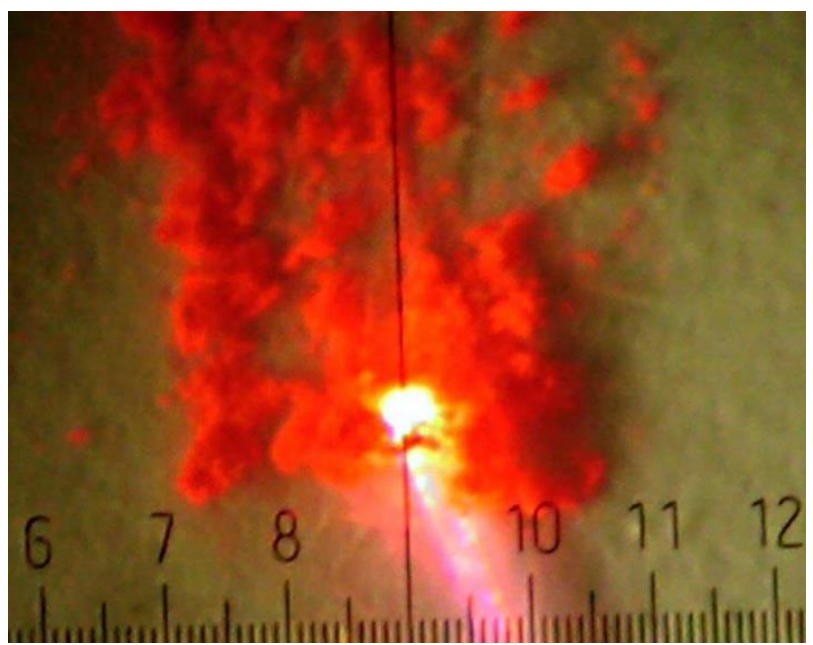

Figure 3A. An example of the use of innovative composite materials-luminophors in laser technology and fiber optics; Red luminophore.

Luminophors shown in Figure 3A and Figure 3B are used in disposable surgical laser instruments.

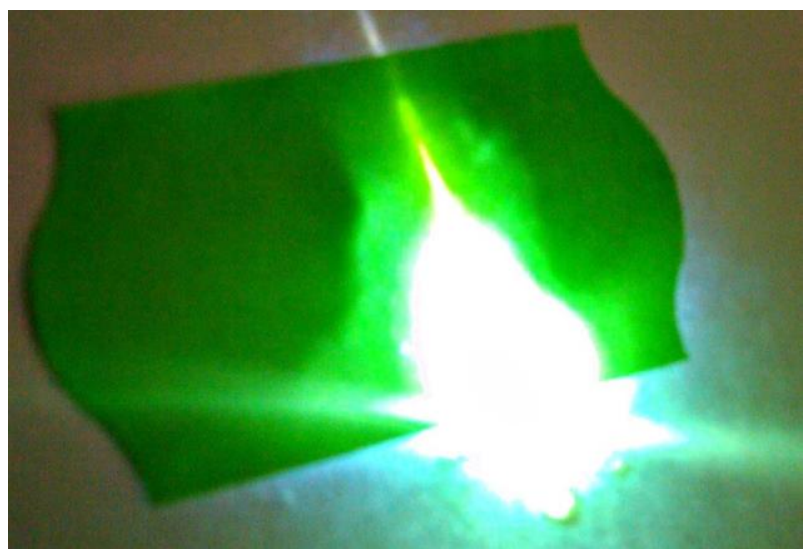

Figure 3B. An example of the use of innovative composite materials - luminophors in laser technology and fiber optics; Green luminophore.

The use of composite materials fundamentally changes even the principles of industrial design - if earlier, when selecting construction materials, the choices made were guided by the materials' properties and parameters, strength, elasticity, chemical resistance, durability, 
specific gravity, electrical resistance, etc. Now when using composite materials, it becomes possible to control the properties and quality of composites, changing the composition of their ingredients and their interrelations.

This is especially noticeable when using carbon-carbon composites. Such composites are produced by multilayer pyrolysis in a vacuum on a substrate of viscose fabric.

The number of pyrolysis layers can be changed depending on the need and because of this, it is possible to define the properties of the material in advance. Thus, the same equipment, the so-called tunnel vacuum furnaces, can produce both flexible and rigid electrodes for electrochemical cells and flexible permeable contacts for electrochemical reactors.

All of the above shows that, on the basis of the same technological methods, it is possible to make various innovative products practically on the same technological equipment.

One of the tasks of the combined software products is targeted coverage. For example, on the website, this could be targeted coverage of all aspects of innovative applications of composite materials and targeted adjustment of output parameters of final products by adjusting the parameters of composites used in their manufacture.

\section{New directions in the technology of manufacturing elements of products and innovative items, including in conditions of smart production}

Technological production lines of modern enterprises are complex and expensive systems and complexes, and each change in production conditions and requirements introduces significant changes and adjustments to the lines. 


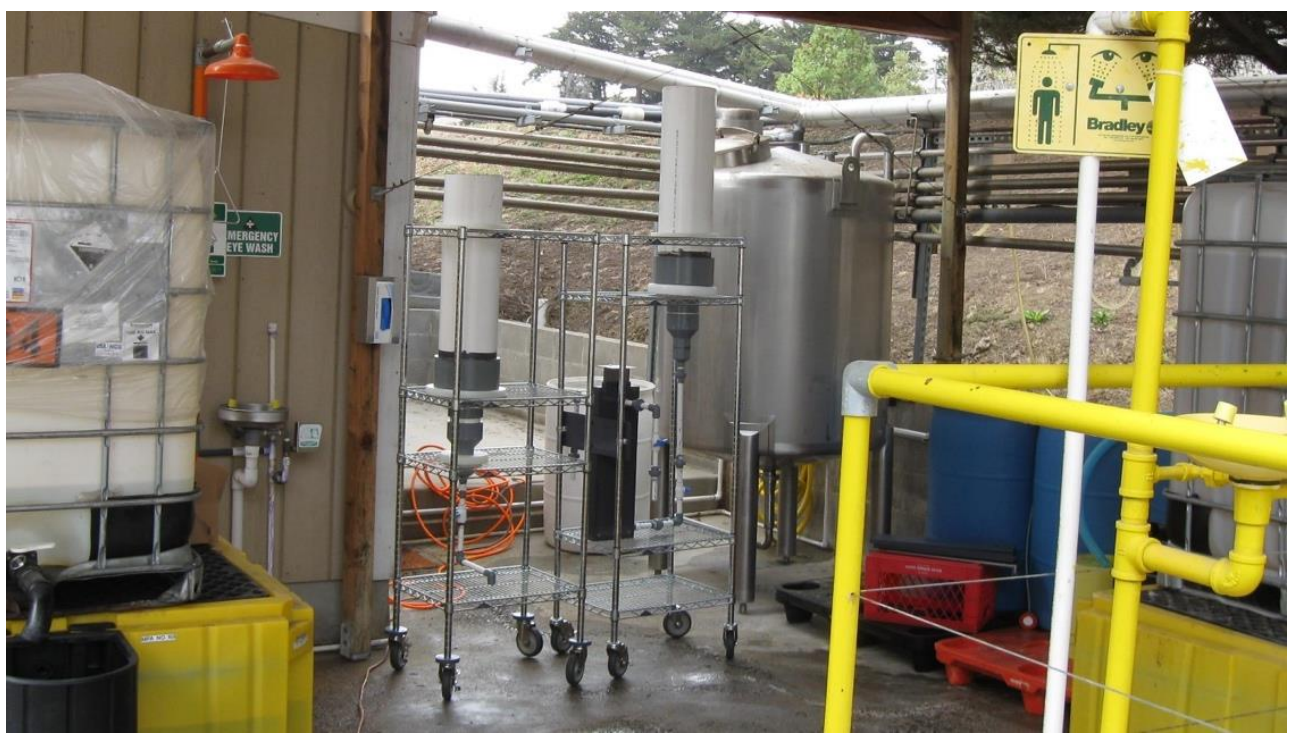

Figure 4. An example of experimental embedding of an innovative system for regeneration of process water without the use of chemical reagents in a typical modern food production facility; an electrochemical reactor which is an integrative invention, is used in the system as the main instrument of electrochemical impact on polluted water

In this situation, the most important thing is not to change the main equipment, and, if possible, to modernize it, embedding new elements into the existing technological line.

In many ways, this possibility of substantial modernization with minimal changes and, accordingly, with minimal costs, determines the reality of the introduction of new products and technologies at existing enterprises.

\section{New consumer standards}




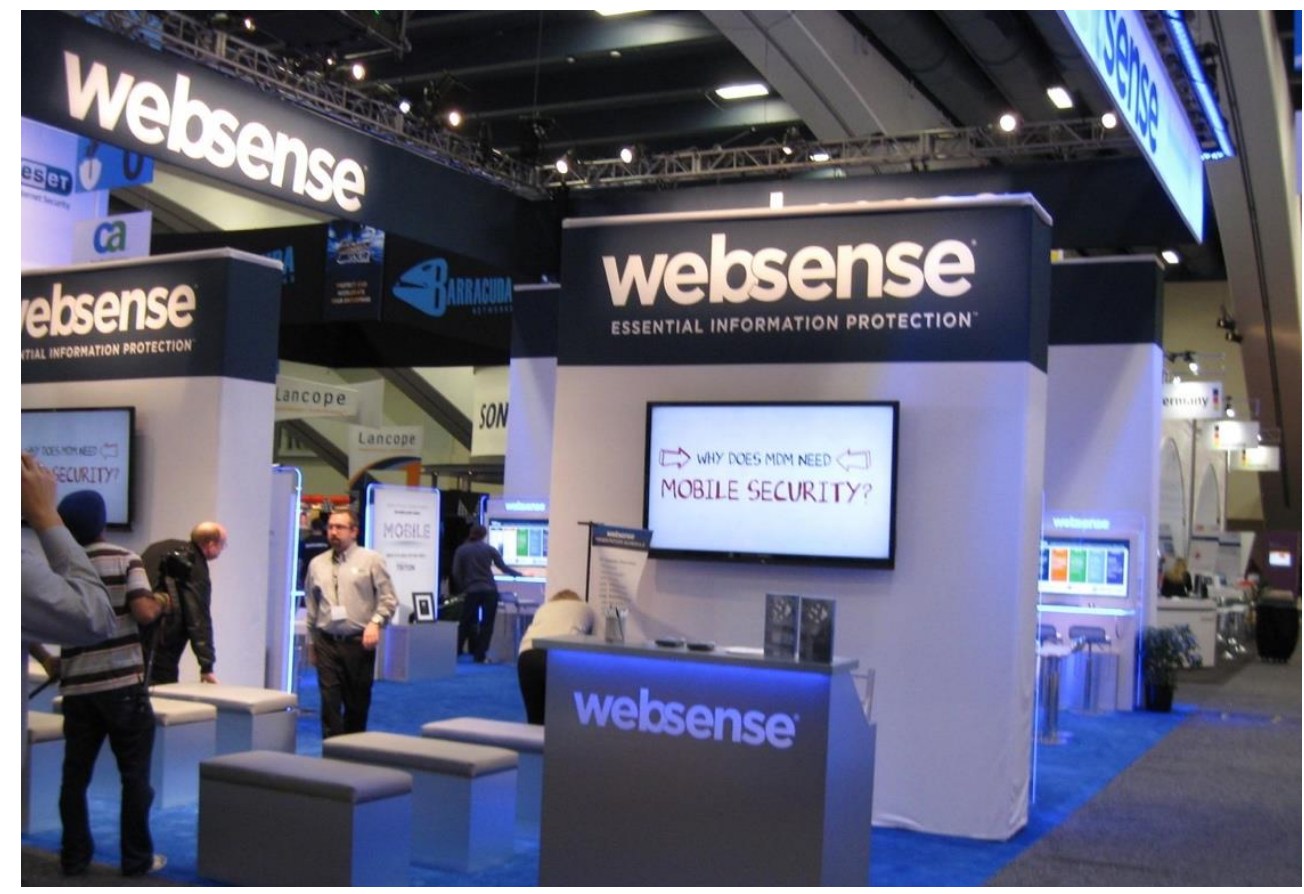

Figure 5. An example of the developers' response to the increased demands of consumers for the level of cyber security, including for web resources

Composite commercial solutions in an innovative economy require an appropriate approach from developers at all levels and especially developers of combined software products, including website developers.

Consumers today focus on ensuring full protection of information, with maximum openness and full clarity of the material presented.

In turn, developers of relevant software products are also interested in the possibility of maximum possible protection of their developments while at the same time maximizing the opportunity to provide users with the fullest informational picture possible.

\section{New environmental standards}




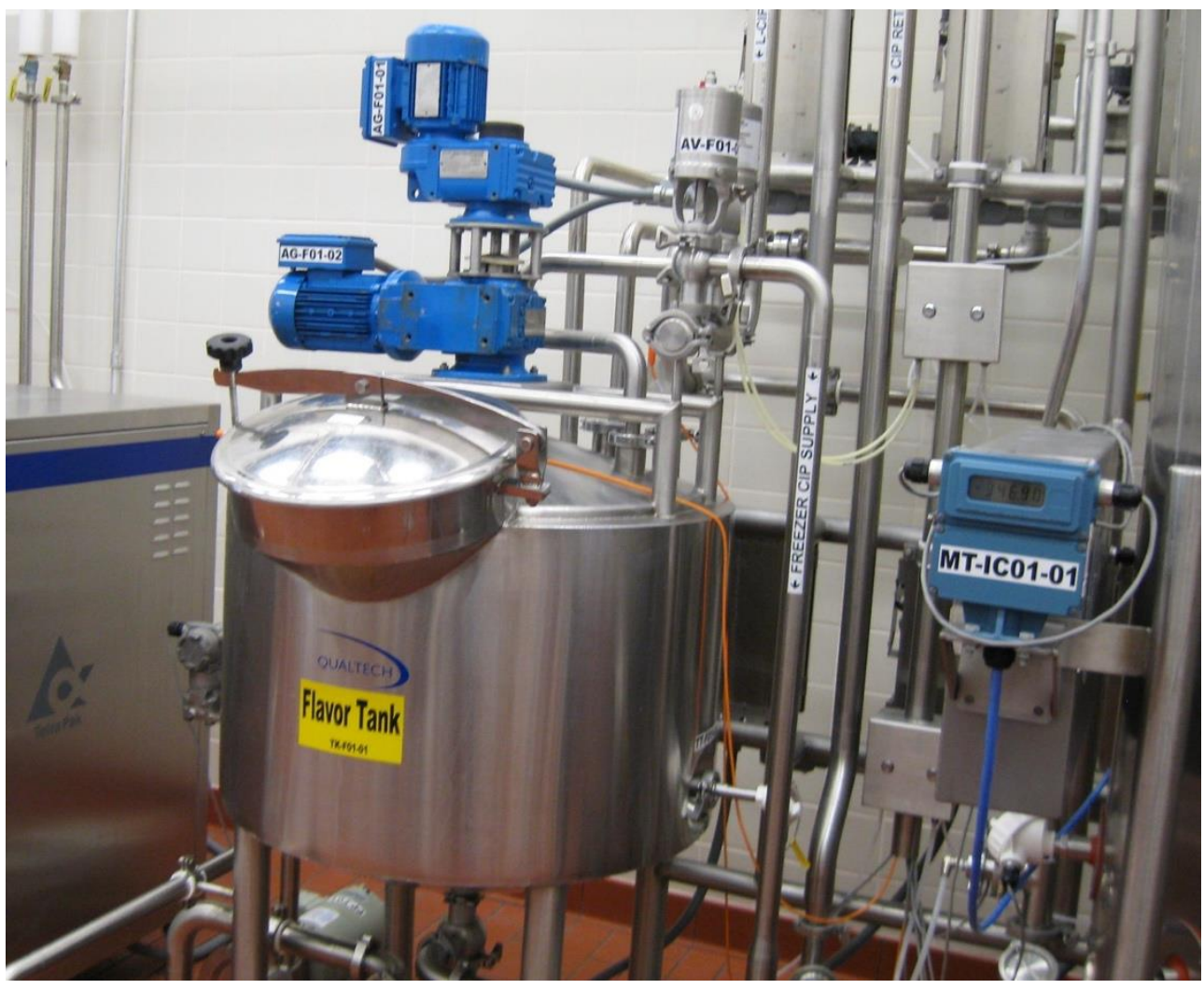

Figure 6. An example of a highly pure and environmentally friendly technological module in an enterprise producing high-quality food; The readers are invited to assess the degree of purity and compliance against the most stringent requirements of environmental standards

Despite the fact that controversial elements are constantly found in the interpretation of requirements and limitations of environmental standards, the control technique itself is constantly being improved. Likewise, the software for consistency and accuracy of control is also being optimized and modified in parallel with the development of new measurement equipment and technology. 


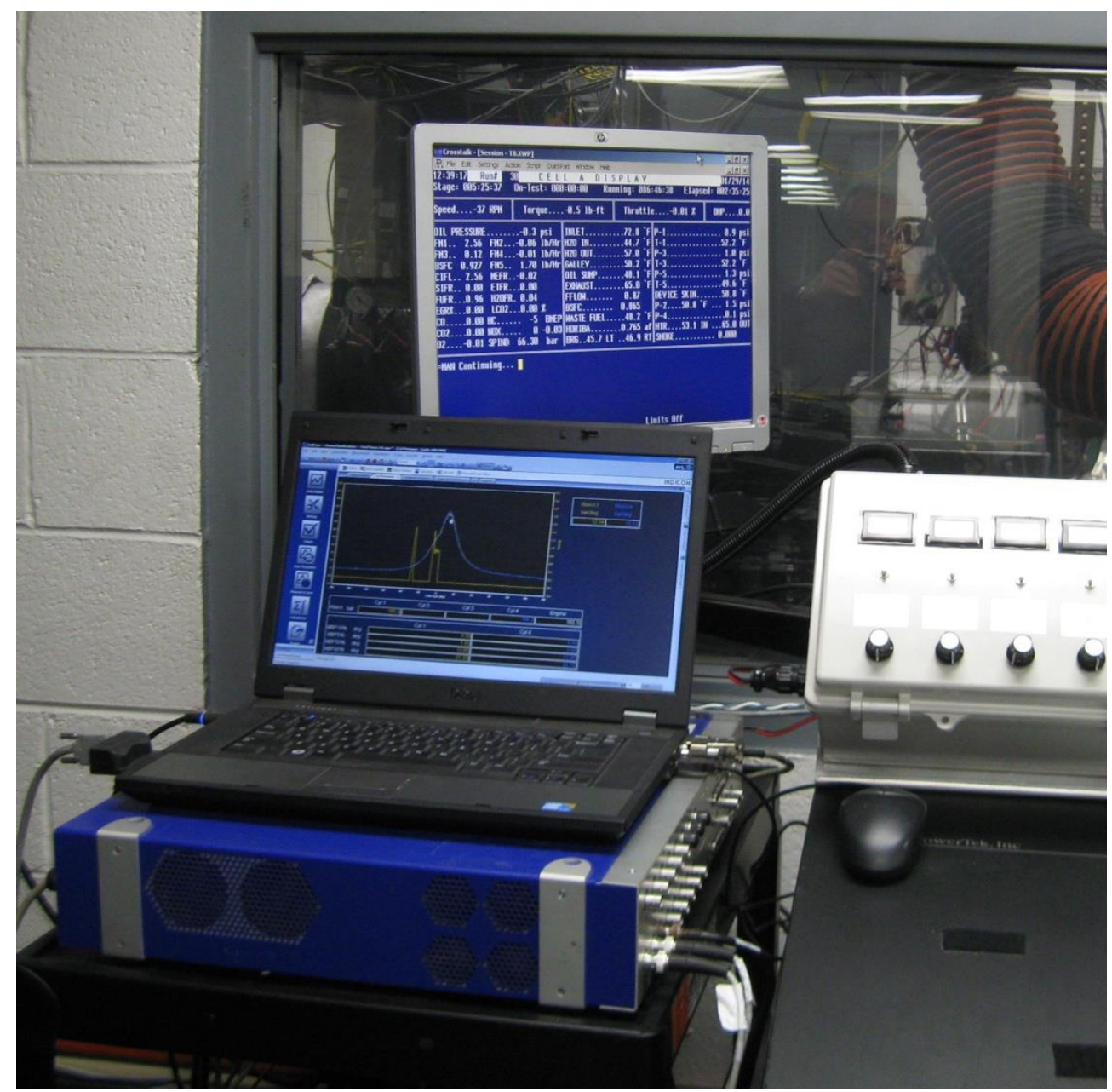

Figure 7. An example of a control system for monitoring the level of toxicity of exhaust gases of a diesel engine that uses diesel fuel of medium viscosity

In countries with a developed innovation economy, maximum attention is paid to the process of the correspondence of environmental standards to real conditions.

Therefore, when presenting, for example, on websites, information on the compliance of technical characteristics and capabilities of new products at the commercialization stages with all limitations of environmental standards, it also requires a preliminary analysis and, to some extent, a forecast of options for making these requirements and values still more stringent.

In the absence of direct options for comparing product indicators at the commercialization stage and tabular values of the restrictive requirements of relevant environmental standards, it makes sense to conduct a system search in specialized laboratories 
of institutes involved in the development of relevant standards and guidance materials and instructions.

\section{Criteria for assessing levels of environmental safety}

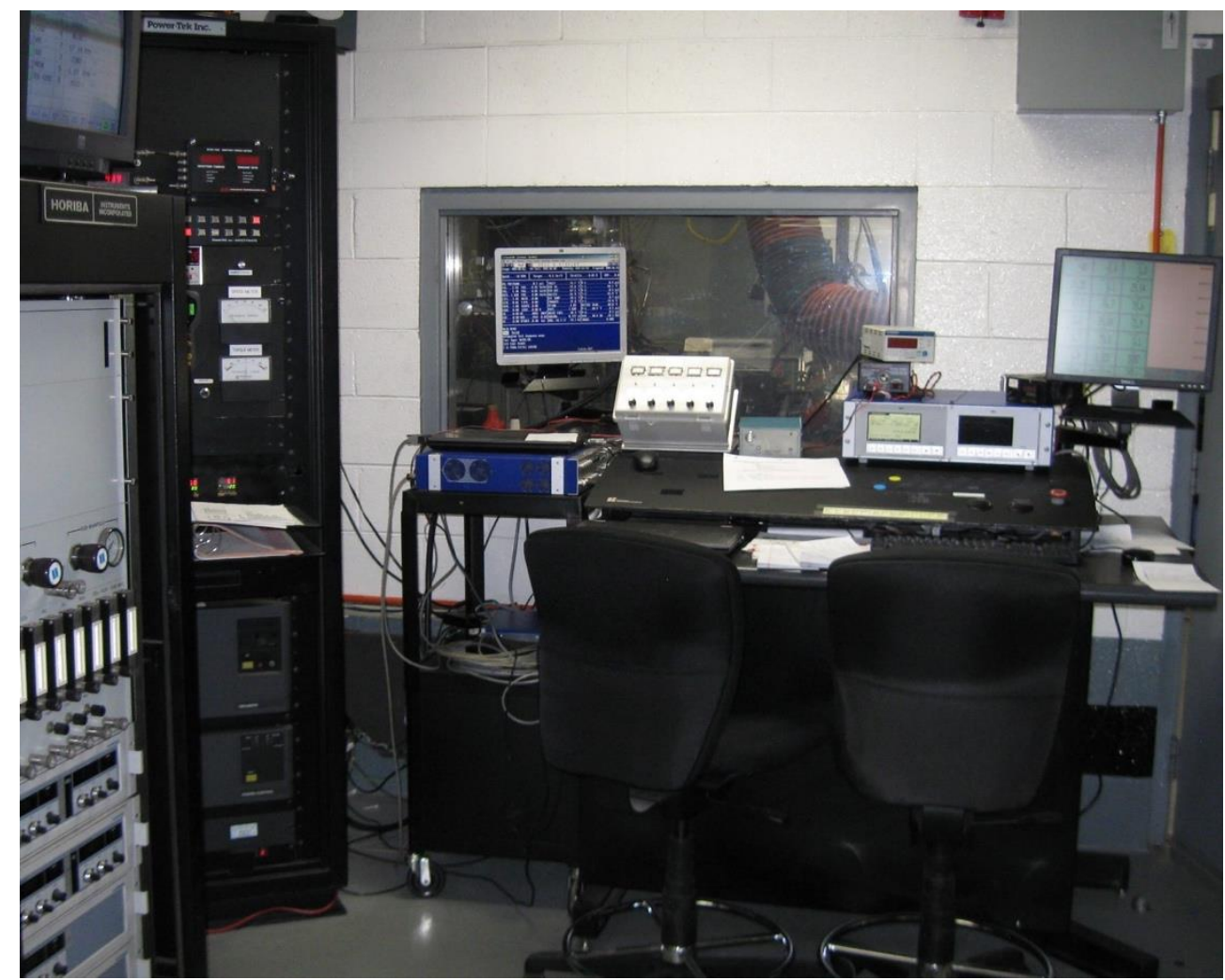

Figure 8. An example of a laboratory complex for analytical verification of compliance of the exhaust gases toxicity level with the requirements and limits of the current environmental standards

As for many product and product groups, indicators of their environmental characteristics are also complex and require, for example, the monitoring of the exhausts of internal combustion engines, and simultaneous monitoring of thousands of parameters. Systematic assessment and comparison of the reference level of environmental safety with actual indicators of a product or item, requires specially developed tables of comparison with 
mathematical models of analytical and comparative evaluation of the same kind of environmental safety to be included in the relevant, official documents.

\section{References}

1. US patent application No20180290403, 11.10.2018. Unitized Composite Structure Manufacturing System / Hasan; Zeaid F.; et al.

2. US patent application No20180285065, 04.10.2018. SMART CONTROLLING DEVICE AND METHOD OF CONTROLLING THEREFOR / JEONG; Gyuhyeok

3. US patent application No20180293778, 11.10.2018. SMART COMPRESSION/DECOMPRESSION SCHEMES FOR EFFICIENCY AND SUPERIOR RESULTS / Appu; Abhishek R.; et al.

4. US patent application No20180285306, 04.10.2018. INTERNET PROTOCOL (IP)ENABLED SMART TRANSDUCER / ESSMANN; ROLAND; et al.

5. US patent application No20180272023, 27.09.2018. SMART OPTIC CONTROLLER FOR A HYDROXYL GENERATOR UNIT / BYSTRZYNSKI; Richard Mariusz; et al.

6. US patent application No20180272657, 27.09.2018. DISPLAY PART PROTECTOR FOR A SMART DEVICE AND METHOD OF ADHERING THE DISPLAY PART PROTECTOR TO THE SURFACE USING THE DEVICE / RYU; JONGYUN; et al.

7. US patent application No20180270076, 20.09.2018. SMART NETWORKING OF TRADITIONAL APPLIANCES / Natarajan; Sreekanth; et al.

8. US patent application No20180270799, 20.09.2018. METHOD AND APPARATUS FOR DOWNLINK CONTROL INFORMATION DESIGN FOR NETWORK COORDINATION / NOH; Hoondong; et al.

9. US patent application No20180293366, 11.10.2018. SYSTEMS AND METHOD FOR SECURELY SHARING AND EXECUTING DATA AND MODELS / Subramaniyan; Arun Karthi; et al.

10. US patent application No20180299849, 18.10.2018. SYSTEMS AND METHODS OF HIERARCHICAL SMART ASSET CONTROL APPLICATION DEVELOPMENT AND OPTIMIZATION / MARTIN; Peter G; et al. 